\title{
Data evaluation on the fly: Auto-Rickshaw at the MX beamlines of the Australian Synchrotron
}

\author{
Santosh Panjikar \\ Australian Synchrotron, ANSTO, Clayton, Australia; \\ santoshp@ansto.gov.au
}

Auto-Rickshaw [1,2] is a system for automated crystal structure determination. It provides computer coded decision-makers for successive and automated execution of a number of existing macromolecular crystallographic computer programs thus forming a software pipeline for automated and efficient crystal structure determination.

Auto-Rickshaw (AR) is freely accessible to the crystallography community through the EMBL-Hamburg AR Server [3].

Recently, it has been installed at the ASCI cluster at the Australian Synchrotron which uses Docker and Kubernetes system for launching AR jobs in high-throughtput manner. The synchrotron AR server is accessible to users from the MX beamline computers.

$A R$ at the MX beamlines can be invoked through command line or a web-based graphical user interface (GUI) for data and parameter input and for monitoring the progress of structure determination. It can be also invoked via automatic data processing if the parameter inputs have been pre set at the AR-GUI during X-ray diffraction experiment.

A large number of possible structure solution paths are encoded in the system and the optimal path is selected as the structure solution evolves. The platform can carry out experimental (SAD, SIRAS, RIP or various MAD) and MR phasing or combination of experimental and MR phasing. The system has extended extensively for evaluation of multiple datasets for various phasing protocols as well as for evaluation of ligand binding and fragment screening.

The new implementation and features will be discussed during the presentation.

[1] Panjikar, S., Parthasarathy, V., Lamzin, V. S., Weiss, M. S. \& Tucker, P. A. (2005). Auto-Rickshaw - An automated crystal structure determination platform as an efficient tool for the validation of an X-ray diffraction experiment. Acta Cryst. D61, 449-457.

[2] Panjikar, S., Parthasarathy, V., Lamzin, V. S., Weiss, M. S. \& Tucker, P. A. (2009). On the combination of molecular replacement and singlewavelength anomalous diffraction phasing for automated structure determination Acta Cryst. D65,1089-1097.

[3] http://www.embl-hamburg.de/Auto-Rickshaw

Keywords: Auto-Rickshaw, Multiple dataset, AR, SAD, MRSAD 